\title{
The wonderful UNESCO collection of Panji tales in Leiden University Libraries
}

\author{
ROGER TOL
}

\begin{abstract}
Leiden University Libraries houses the greatest collection of Panji manuscripts in the world. This became evident while preparing the successful nomination of Panji tales manuscripts for UNESCO's Memory of the World Programme. This article begins with observations on Panji tales in general. They originated in East Java and have subsequently spread to other areas in Southeast Asia. This is followed by a description of the collection's composition and its history. The collection exists of over 260 manuscripts in eight languages, the majority of manuscripts written on palm leaf or paper. I have described four manuscripts in detail paying special attention to their provenance and history. Originating from four locations, written in four languages in three different scripts, they can be considered representative of the collection. A complete listing of all manuscripts is given in the appendix. ${ }^{1}$
\end{abstract}

KEYWORDS

Panji tales; manuscripts; history of manuscripts; Memory of the World.

\section{INTRODUCTION}

Panji tales manuscripts were inscribed in UNESCO's Memory of the World register in 2017. It had taken several years of great effort by a number of individuals and institutions to prepare. Apart from Leiden University Libraries, from the early stages on the national libraries of Indonesia,

1 Thanks to an equally wonderful initiative from Leiden University Libraries, the collection of Panji manuscripts can now also be consulted online: https:/ / digitalcollections.universiteitleiden. $\mathrm{nl} /$ panji. This site will be the inevitable starting point for all of the upcoming Panji research.

ROGER TOL (1950), was the director of KITLV-Jakarta from 2003 until his retirement in 2015. From 1993 to 2003 he was Chief Librarian of the KITLV/Royal Netherlands Institute of Southeast Asian and Caribbean Studies in Leiden, the Netherlands. Roger Tol received his PhD from Leiden University in 1989 with a dissertation on a Buginese historical poem. His main research interests are in Buginese and Malay traditional texts. Roger Tol may be contacted at: roger.tol@gmail.com. 
Malaysia, and Cambodia also participated in the nomination. ${ }^{2}$ As a person closely associated with the whole process for Leiden University Libraries, I have made an inventory of all Panji tales manuscripts in their collection. This was a time-consuming task since its collection of oriental manuscripts is simply overwhelming - the result of an active acquisition policy that has been in place for more than 400 years. The libraries' collection programme has always covered a very wide spectrum of the humanities, which has led to a huge quantity of Asian and Southeast Asian manuscripts, including from the Indonesian archipelago. Among the many genres and languages in the collection, we also find old and new texts which are known as Panji tales. A systematic search for all Panji tales in this collection had never been undertaken before.

After consulting all available databases and catalogues, I found more than 260 manuscripts - while I shall have surely missed a manuscript or two. ${ }^{3}$ They use a variety of materials, most of them on European paper and palm leaf, and are written in eight Indonesian languages. A full listing is presented in the Appendix. No doubt this constitutes the greatest collection of Panji manuscripts in the world.

\section{PANJI TALES: What ARE THEY?}

Panji stories relate the adventures of Prince Panji Raden Inu Kertapati and his sweetheart, Candra Kirana, who have been separated and must overcome many intricate hurdles before managing to reunite. There are many versions of this core tale with variations in locations, persons, and names. Metamorphoses, disguises, sex changes, and all sorts of supernatural events abound in the narratives. They are indeed essential elements of Panji tales.

From the earliest beginning of scholarly interest in Indonesia, Panji tales have been subject to academic studies because of their attractive literary and referential narratives. Apart from influencing many oral and written literatures, Panji tales have also been influential in performing arts like shadow plays (wayang) and dance drama like gambuh on Bali.

Originating in East Java, the tales have been spread throughout Southeast Asia. They are found in at least twelve languages with Javanese-Balinese, Javanese, and Malay the most numerous. But there are also texts in Balinese, Sasak (Lombok island), Buginese (South Sulawesi), Acehnese (North Sumatra), Sundanese (West Java), Thai, Vietnamese, Burmese (Myanmar), and Khmer (Cambodia). Panji manuscripts are kept in many major public and private collections all over the globe. This is a sign of their importance and international influence.

2 The nomination form is available online (without the appendixes) at http:/ / www.unesco. $\mathrm{org} / \mathrm{new} / \mathrm{en} / \mathrm{communication}$-and-information/memory-of-the-world/register/full-list-ofregistered-heritage/registered-heritage-page-7/ panji-tales-manuscripts/.

3 The following sources have been consulted: Brandes 1901-26; Ekadjati 1988; Iskandar 1999; Juynboll 1899, 1907, 1912; Marrison 1994; Matthes 1875; Pigeaud 1967-80; Pijnappel 1871a, 1871b; Voorhoeve 1994; Vreede 1892; Wan Ali 1985; Wieringa 1998, 2007; Witkam 2006-. 
Panji tales are an excellent example of global mythical tales with a specific local content. Through its main theme, the intricate and utmost perilous quest undertaken by a prince to win his princess, it is evidently a part of mythic global narratives like those found in the Indian and western world. Likewise, its local features clearly show aspects of many Austronesian myths of origin.

\section{PANJI TALes IN LeIDEN University LibRARIES}

Since its establishment in 1575, the library of Leiden University has collected reading materials for its students and lecturers. When the Netherlands became involved in different parts of Asia at the beginning of the seventeenth century, manuscripts were also obtained over there and ended up in Leiden via various routes in space and time. The Dutch presence in Asia marked the beginning of a continuous acquisition policy which has lasted over 400 years. Obviously, Indonesia has been of the greatest interest in this respect and over the centuries there has been a steady and voluminous flow of Indonesian manuscripts to enrich the library's collection. Indonesian manuscripts now amount to over 10,000 codices in many languages of the archipelago.

\section{Characterization OF THE COllection}

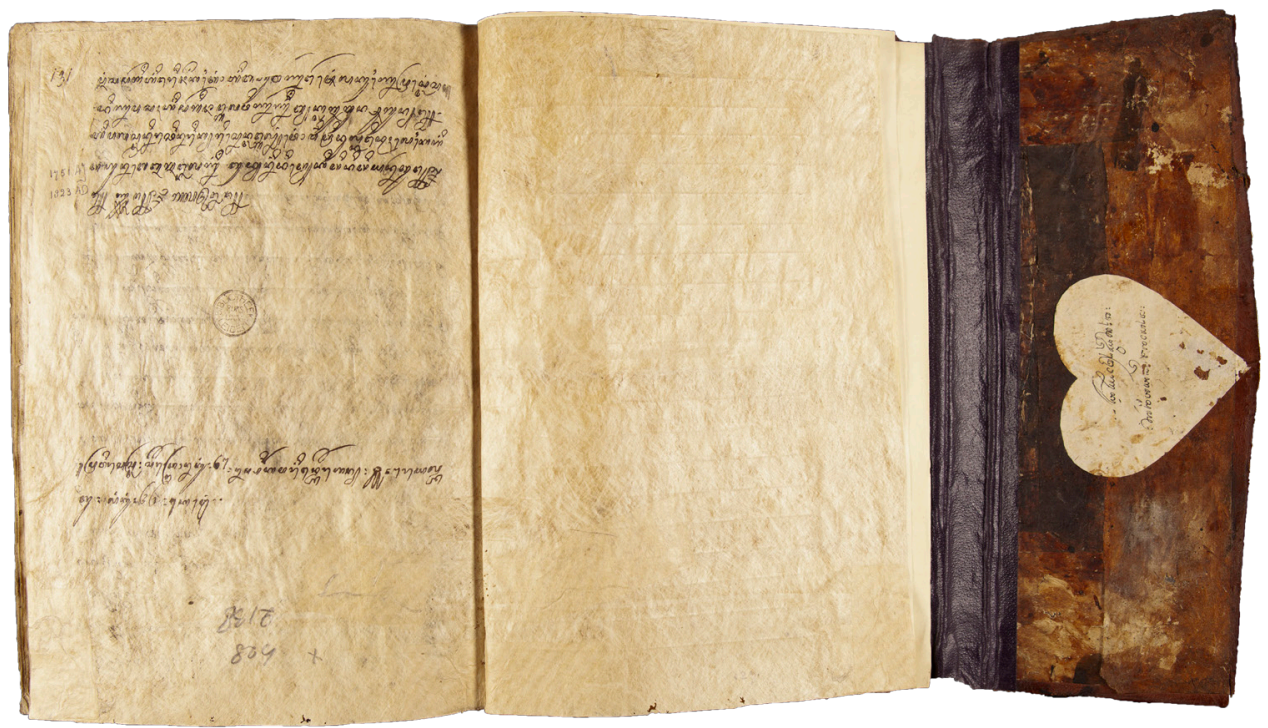

Illustration 1. Panji Ngron Akun. East-Javanese manuscript from 1823, written on daluang. (Cod.Or. 2138 (2)).

The collection displays an extraordinarily broad scope in languages, geographical spread, materials, and size. There are Panji tales in at least eight languages: Javanese-Balinese, Javanese, Malay, Balinese, Sasak, Sundanese, Acehnese, and Buginese. ${ }^{4}$ These languages correspond to the geographical 
spread of the manuscripts with most of them originating from the islands of Java and Bali, but also from the northern tip of Sumatra, the island of Lombok, and South Sulawesi.

Most of the manuscripts have been written on either palm leaf (lontar) or (European) paper. A few scribes have used tree bark (daluang) as their medium; see Illustration 1.

Some of the manuscripts are very voluminous, like the three-volume Javanese Panji Jaya Lengkara, totalling a staggering 1,275 beautifully written pages. ${ }^{5}$ Others are obviously fragments of the story and contain only a few pages or leaves.

\section{EACH MANUSCRIPT HAS ITS OWN STORY}

A manuscript can travel many routes to a library collection. Some are bought from private persons or at auctions, others donated by individuals or institutions. These are the final episodes in a manuscript's life and, in all modesty, not very exciting. The birth of a manuscript, however, and its life before it arrival at its final resting place can be quite extraordinary. They might have been valued as treasures and treated as heirlooms. They could have been read aloud at special ceremonies or on other solemn occasions. Maybe they have been used as instruments for healing the sick or to interpret and understand natural phenomena. Or they have led a quiet life, read only once by some scholar or never even read or used at all. All of them might also have faced natural disasters, revolutions and war.

Let us see how four of the Panji manuscripts at Leiden University Libraries have fared. Originating from four locations, written in four languages in three different scripts, they can be considered representative for the collection.

\section{Manuscript Cod.Or. 3721 (see Illustration 2)}

In the year 1725 on the island of Bali, the 109 palm leaves of this manuscript were incised and rubbed with soot to make the letters visible. The palm leaves were pressed between bamboo covers - one original bamboo cover is still there, the other is missing. It is written in the Javanese-Balinese language using the Balinese script and contains a Malat text. ${ }^{6}$

Manuscript Cod.Or. 3721 is the oldest known Malat manuscript in existence. The leaves of this manuscript have darkened and have been soiled with some superficial grime, but the text is generally still legible. It is written in the Balinese district of Mengwi, probably the village of Bongkasa. ${ }^{7}$ It was very

distinct from - Old-Javanese. Javanese-Balinese is different from both the Javanese and the Balinese language.

5 Cod.Or. 1871, written in 1830. It was copied for C.F. Winter (1799-1859) from a manuscript in the library of the Susuhunan of Surakarta.

$6 \quad$ In Bali Malat texts are the most popular and voluminous Panji tales and are composed in tengahan metres. Apart from Brandes MS 54 in the National Library of Indonesia, no manuscripts containing the complete Malat text are still in existence.

7 The colophon reads "the west of the kingdom of Bali, during a war, without a place to 
probably then kept in the nearby palace of Kaba-Kaba until it was acquired by the famous linguist and specialist of Austronesian languages Herman Neubronner van der Tuuk (1824-1894). Van der Tuuk lived and worked in Bali from 1870 until his death in 1894, so the manuscript must have come into his possession in these years. According to a note by Van der Tuuk on the front leaf, he purchased the manuscript from a person named I Made Karang for the price of three Spanish dollars ("3 sp. matte"); see Illustration 3. As his name shows, this I Made Karang was a commoner, that is not of royal descent.

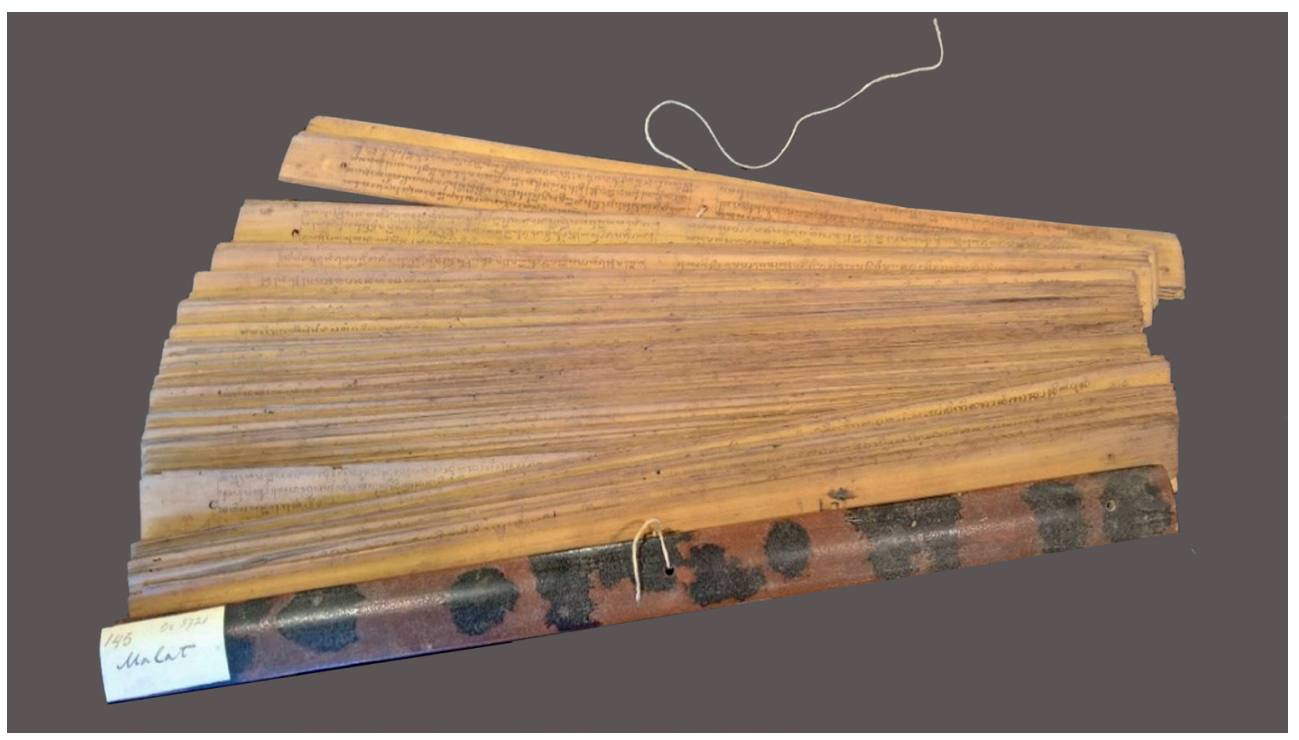

Illustration 2. Malat. Dating from 1725 this is the oldest Malat text in existence. Written on palm leaf, from Bali. (Cod.Or. 3721).

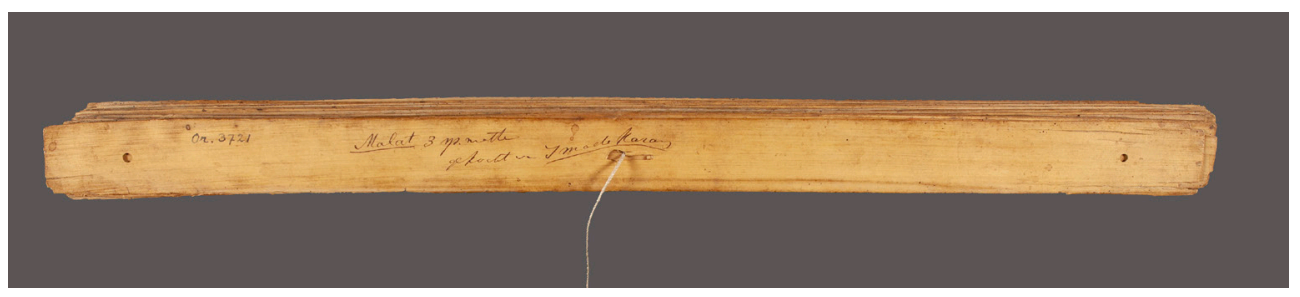

Illustration 3. Note by H.N. van der Tuuk stating he purchased the manuscript from I Made Karang for 3 Spanish dollars. (Cod.Or. 3721).

Under the terms of his will, after Van der Tuuk's demise in 1894 his collection of manuscripts and books were to be sent to Leiden University Libraries. However, they were first sent to Batavia for inventorization and cataloguing. There a four-volume catalogue of his manuscripts was prepared

shelter; to the east of Liring Anggora south of the four small streams". This points to the village of Bongkasa, which is situated to the north of Mengwi (Vickers 2005: 88). 
by J.L.A. Brandes in which our manuscript appears as no. 633 (Brandes 1901-26, III: 143). Finally, in 1896 the manuscript entered Leiden University Libraries, where it received its current shelf mark Cod.Or. 3721.

\section{MAnuscript CoD.Or. 1825 (SEe Illustration 4)}

This beautiful manuscript was written in the Javanese language using Javanese script. The text has been given the title of Murta Smara. Written on European (Dutch) handmade paper, it is 306 pages long and bound in a European-style binding, with a half leather and marbled paper board covering.

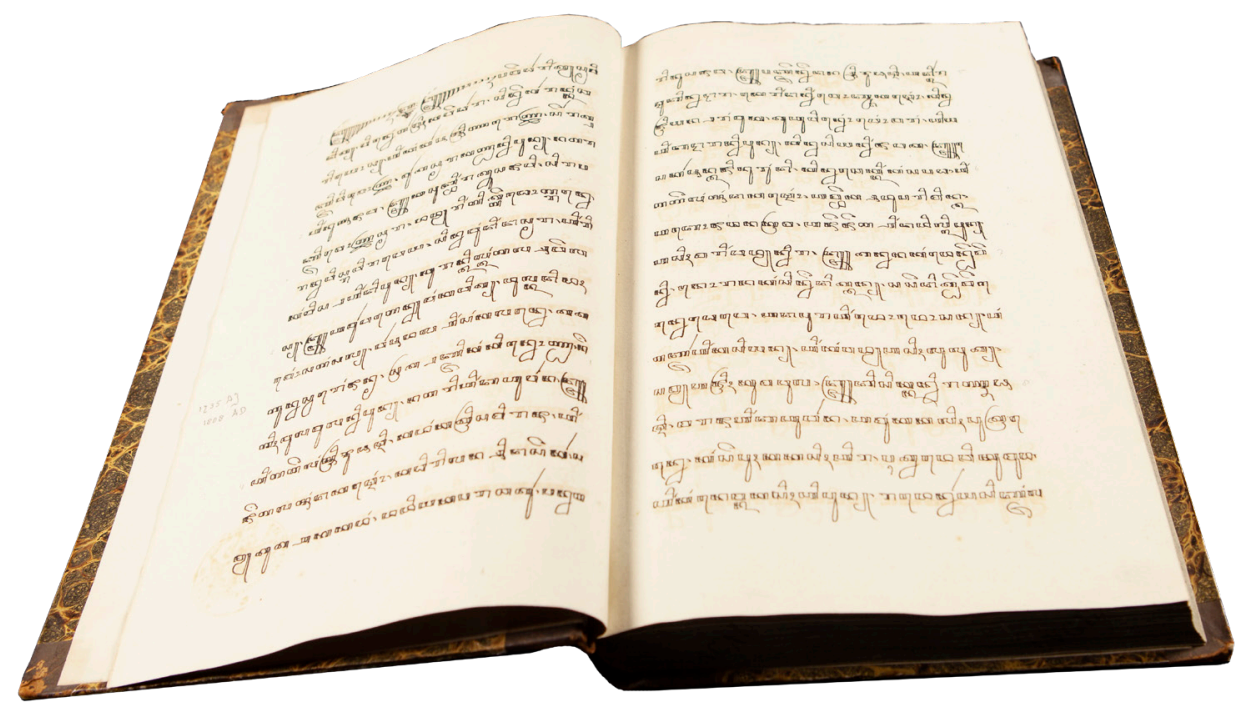

Illustration 4. Murta Smara. Javanese, Javanese script, written in Surakarta in 1808 on European handmade paper. (Cod.Or. 1825).

The manuscript itself states that it was written in the kingdom of Surakarta in 1808 but then we lose track for around forty years. At a certain moment, it made the journey to the Netherlands, because it shows up as belonging to the collection of the Royal Academy in Delft, an institution for the training of civil servants. This academy flourished from 1842 to 1864 . After its closure in 1864, our manuscript - along with all other manuscripts of the Royal Academy - travelled a mere 26 kilometres north to enter the collection of Leiden University Libraries.

Leiden University Libraries possesses an important supporting document which contains a complete romanized transcription of manuscript Cod.Or. 1825 made by Th.G.Th. Pigeaud (1899-1988), the eminent scholar of Javanese. It is kept as manuscript Cod.Or. 6751 with the title "Serat Pandji Moertasmara".

\section{Manuscript Cod.Or. 1709 (see Illustration 5)}

This Panji tale was written in the Malay language using a modified Arab script (jawi) in 1821. It contains the Hikayat Cekel Waneng Pati, the story of 
Cekel Waneng Pati, one of the names of Prince Panji. The text is written on handmade European paper with a length of 183 pages, now enclosed in a twentieth-century repair binding of another European half-linen binding.

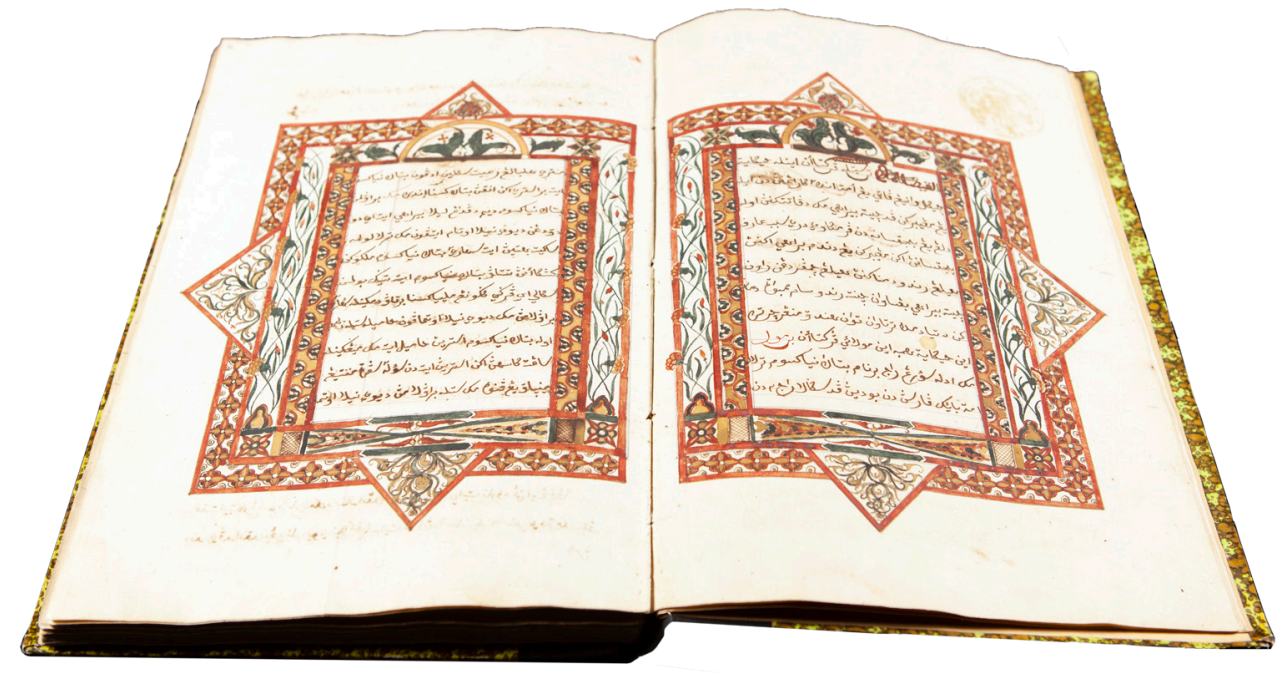

Illustration 5. First two illuminated pages. Hikayat Cekel Waneng Pati. Malay, written in jawi script in 1821 on European handmade paper. (Cod.Or. 1709).

This manuscript was first mentioned by the famous linguist H.N. van der Tuuk (1824-1894) in his "Short account of the Malay manuscripts in the Royal Asiatic Society in London", published in 1866 (Van der Tuuk 1866). On page 429 he describes two manuscripts of the Hikayat Cekel Waneng Pati. He writes (in English translation): "The text of the first number is similar to a manuscript belonging to the Ministry of Colonial Affairs in The Hague (a folio of 185 pages and marked: Ned. Kolonien. Handschriften $C n^{\circ}$. 21; ends abruptly), but is more extensive".

Unquestionably this remark refers to our manuscript Cod.Or. 1709 since, apart from having the same number of pages, the words Ned. Kolonien. Handschriften $C n^{\circ} .21$ are still there on page 197; see Illustration 6. His remark also clarifies the history of the manuscript: most probably composed at the Government General Secretariat in Batavia for educational purposes, it moved to Ministry of Colonial Affairs in The Hague, then to the Delft Academy, before it landed in Leiden in 1864 and was given the shelf mark Cod.Or. 1709.

Manuscript Cod.Or. 1709 has been used in several important academic studies, like W.H. Rassers (1877-1973) in his dissertation "De Pandji-roman" (The Panji novel) (Rassers 1922: 18-54). ${ }^{8}$

8 In an article devoted to Rassers dissertation, J.J. Ras (1926-2003), provides an abridged English translation of Rassers' summary of Cod.Or. 1709 (Ras 1973: 416-418). Other references to this manuscript in Winstedt 1969: 55; Liaw Yock Fang 2011: 150, 153; Iskandar 1995: 225-226 (with a Malay summary of the contents of Cod.Or. 1709); Braginsky 2004: 139-140. 


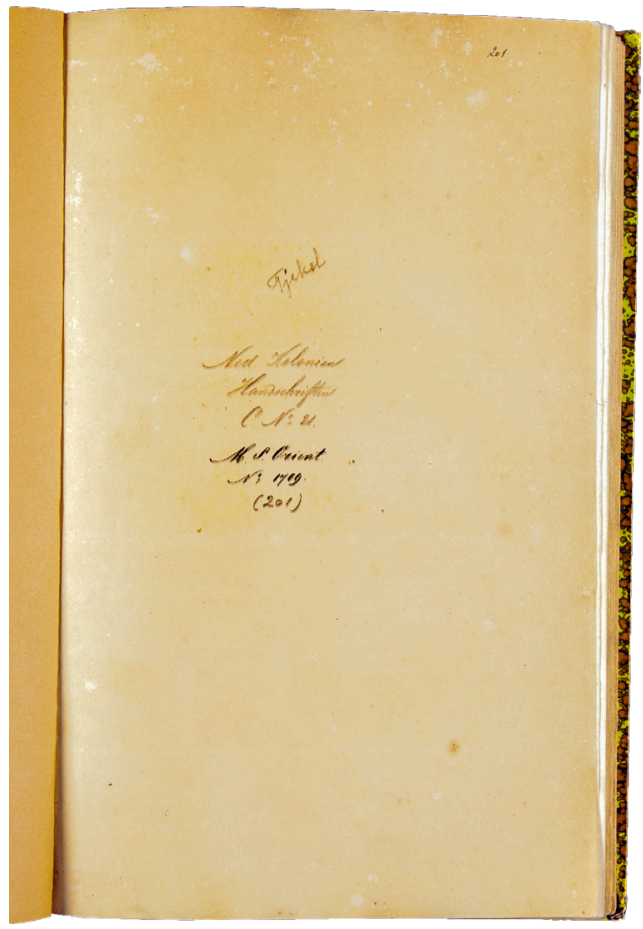

Illustration 6. Former and current shelf marks. (Cod.Or. 1709, p. 197).

4. MANuscript Cod.Or. 8102 (SEe Illustration 7)

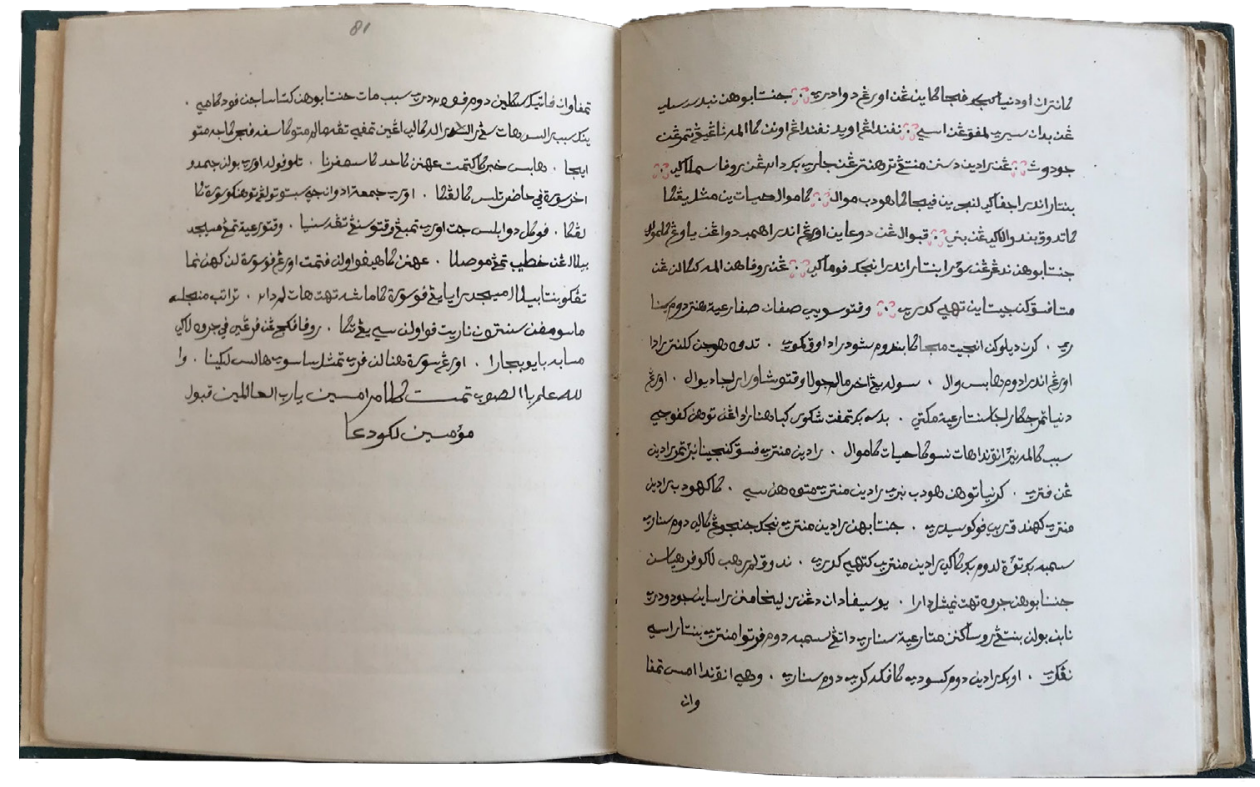

Illustration 7. Hikayat Cintabuhan. Achehnese in jawi script; 1872. From the Snouck Hurgonje collection. (Cod.Or. 8102, pp. 80-81). 
Panji tales also found their way to Aceh, the former sultanate located at the northern tip of the island of Sumatra, and for many centuries a centre of Islamic scholarship. This fine, well-preserved manuscript containing the Acehnese Hikayat Cintabuhan bears witness to Panji's wanderings to "The veranda of Mecca", as Aceh was known. With its 81 quarto-sized pages, it is a relatively small manuscript; but it contains a complete text, not a fragment.

Written in 1872, it ended up in the library by way of Christiaan Snouck Hurgonje (1857-1936), the famously influential orientalist, scholar of Arabic and Islam, and expert on Aceh. He had spent from July 1891 to February 1892 in Aceh and most probably this manuscript came into his possession during this time (Voorhoeve 1994: 15). When Snouck Hurgronje left the Indies in 1906, he took his collection of books and manuscripts with him to Leiden where he lived until his death. After having given his collection of Oriental manuscripts in permanent loan to the Leiden University Library as early as 1907, he bequeathed his collection of printed books and Oriental manuscripts to the library at his death in 1936 (Voorhoeve 1994: 16; Vrolijk 2013).

In his survey of Acehnese literature in The Achehnese 9 Snouck Hurgronje refers to this text as follows:

Chintabuhan is the Malay Kén Tambuhan or Tabuhan; by and large the Achehnese romance corresponds to Klinkert's edition of the Malay poem of that name [=Klinkert 1886].

In the Achehnese hikayat the princess's country is called Tanjong Puri and she is not borne away to the forest by supernatural force as in the Malay tale, but carried off by Radén Meuntròë's own father who declares war on her sire for refusing to pay him tribute.

The Achehnese composer has also given a slightly Mohammadan tinge to the whole. The diwas [these are dewa's, 'gods'], it is true, play a weighty part and work all manner of marvels, but not until Allah has expressly charged them so to do; and people in distress invoke the aid, not of the all administering diwas, but of the Almighty Creator. (Snouck Hurgonje 1906, Vol. 2: 149).

\section{REFERENCES}

Braginsky, V. 2004. The heritage of traditional Malay literature; A historical survey of genres, writings and literary views. Leiden: KITLV Press. [Verhandelingen van het Koninklijk Instituut voor Taal-, Land- en Volkenkunde 214.]

Brandes, J.L.A. 1901-26. Beschrijuing der Javaansche, Balineesche en Sasaksche handschriften aangetroffen in de nalatenschap van Dr. H. N. van der Tuuk, en door hem vermaakt aan de Leidsche Universiteitsbibliotheek. 4 vols. Batavia [et cetera]: Landsdrukkerij.

Ekadjati, E (ed.). 1988. Naskah Sunda; Inventarisasi dan pencatatan. Bandung/ Tokyo: Lembaga Penelitian Universitas Padjadjaran; Toyota Foundation. Iskandar, T. 1995. Kesusasteraan klasik Melayu sepanjang abad. Brunei: Jabatan Kesusasteraan Melayu, Universiti Brunei Darussalam. 
Iskandar, T. 1999. Catalogue of Malay, Minangkabau, and South Sumatran manuscripts in the Netherlands. 2 vols. Leiden: Documentatiebureau IslamChristendom.

Juynboll, H.H. 1899. Catalogus van de Maleische en Sundaneesche handschriften der Leidsche Universiteits-Bibliotheek. Leiden: Brill.

Juynboll, H.H. 1907. Supplement op den catalogus van de Javaansche en Madoereesche handschriften der Leidsche Universiteits-Bibliotheek. Dl. 1: Madoereesche handschriften, Oudjavaansche inscripties en Oud-en Middeljavaansche gedichten. Leiden: Brill.

Juynboll, H.H. 1912. Supplement op den catalogus van de Sundaneesche handschriften, en Catalogus van de Balineesche en Sasaksche handschriften der Leidsche Universiteits-Bibliotheek. Leiden: Brill.

Klinkert, H.C. 1886. Drie Maleische gedichten, of De sjaïrs Ken Tamboehan, Jatim Noestapa en Bidasari. Leiden: Brill.

Liaw Yock Fang. 2011. Sejarah kesustraan Melayu klasik. Jakarta: Yayasan Pustaka Obor Indonesia. [First edition 1975.]

Marrison, G.E. 1994. The literature of the Sasak of Lombok; A survey of Javanese and Sasak texts. [Hull]: Centre for South-East Asian Studies, University of Hull.

Matthes, B.F. 1875. Kort verslag aangaande alle mij in Europa bekende Makassaarsche en Boeginesche handschriften, vooral die van het Nederlandsch Bijbelgenootschap te Amsterdam. Amsterdam: Nederlandsch Bijbelgenootschap.

Pigeaud, Th.G.Th. 1967-80. Literature of Java; Catalogue raisonné of Javanese manuscripts in the Library of the University of Leiden and other public collections in the Netherlands. 4 vols. Lugduni Batavorum (Leiden): In Bibliotheca Universitatis.

Pijnappel, J.Gz. 1871a. "De Maleische handschriften der Leidsche bibliotheek", Bijdragen tot de Taal-, Land-en Volkenkunde 18: 142-148.

Pijnappel, J.Gz. 1871b. “Catalogus der Maleische handschriften in de bibliotheek der Leidsche Akademie", Bijdragen tot de Taal-, Land-en Volkenkunde 18: 149-178.

Ras, J.J. 1973. “The Panji romance and W.H. Rassers' analysis of its theme”, Bijdragen tot de Taal-, Land-en Volkenkunde 129: 411-456.

Rassers, W.H. 1922. De Pandji-roman. Antwerpen: De Vos - Van Kleff.

Snouck Hurgronje, C. 1893-94. De Atjèhers. Batavia: Landsdrukkerij; Leiden: Brill.

Snouck Hurgronje, C. 1906. The Achehnese. Translated by A.W.S. O'Sullivan. Leyden: Brill.

Tuuk, H.N. van der. 1866. “Kort verslag der Maleische handschriften, toebehoorende aan de Royal Asiatic Society te Londen", Bijdragen tot de Taal-, Land- en Volkenkunde 13: 409-461.

Vickers, A. 2005. Journeys of desire; A study of the Balinese text Malat. Leiden: KITLV Press. [Verhandelingen van het Koninklijk Instituut voor Taal-, Land- en Volkenkunde 217.]

Voorhoeve, P. 1994. Catalogue of Acehnese manuscripts in the Library of Leiden University and other collections outside Aceh. Leiden: Leiden University Library (Legatum Warnerianum). [Codices manuscripti 24.] 
Vreede, A.C. 1892. Catalogus van de Javaansche en Madoereesche handschriften der Leidsche Universiteits-bibliotheek. Leiden: Brill.

Vrolijk, Arnoud. 2013. Collection Christiaan Snouck Hurgronje; Collection guide. [Version update: 3 December 2013, http:/ / hdl.handle.net/1887.1/ item:1887407; accessed 8-11-2018.]

Wan Ali. 1985. Katalog manuskrip Melayu di Belanda = Catalogue of Malay manuscripts in the Netherlands. Kuala Lumpur: Perpustakaan Negara Malaysia. [Siri Bibliografi Manuskrip No. 1.]

Wieringa, E.P. 1998. Catalogue of Malay and Minangkabau manuscripts in the library of Leiden University and other collections in the Netherlands. Leiden: Leiden University Library (Legatum Warnerianum).

Wieringa, E.P. 2007. Catalogue of Malay and Minangkabau manuscripts in the library of Leiden University and other collections in the Netherlands. Vol. 2: Comprising the H.N. van der Tuuk bequest acquired by the Leiden University Library in 1896. Leiden: Leiden University Library.

Winstedt, R. 1969. A history of classical Malay literature. Kuala Lumpur [et cetera]: Oxford University Press. [Oxford in Asia historical reprints.]

Witkam, J.J. 2006-. Inventory of the Oriental manuscripts in Leiden University Library. [Http:/ / www.islamicmanuscripts.info/inventories/leiden/ index.html; accessed 18-9-2018.] 
APPENDIX:

List of PANJI-TALES MANUSCRIPTS IN LEIDEN UNIVERSITY LIBRARIES

\begin{tabular}{|c|c|c|c|c|}
\hline & $\begin{array}{l}\text { Codex number } \\
\text { (Cod.Or.) }\end{array}$ & Title & Language & Date \\
\hline 1 & Cod.Or. 1709 & $\begin{array}{l}\text { Hikayat Cekel } \\
\text { Wanengpati }\end{array}$ & Malay & 1821 \\
\hline 2 & Cod.Or. 1755 & $\begin{array}{c}\text { Hikayat Susunan } \\
\text { Kuning Dalam Negeri } \\
\text { Gagelang. }\end{array}$ & Malay & 1820 \\
\hline 3 & Cod.Or. 1765 & $\begin{array}{l}\text { Syair Undakan } \\
\text { Agung Udaya }\end{array}$ & Malay & 19th century \\
\hline 4 & Cod.Or. 1766 & [Panji syair] & Malay & 19th century \\
\hline 5 & Cod.Or. 1770 & Surat Gambuh. syair & Malay & 19th century \\
\hline 6 & Cod.Or. 1778 & $\begin{array}{l}\text { [fragment of a Panji } \\
\text { story] }\end{array}$ & Malay & 19th century \\
\hline 7 & Cod.Or. 1816 & Kuda Narawangsa & Javanese & 1818 \\
\hline 8 & Cod.Or. 1825 & Murta Smara & Javanese & 1808 \\
\hline 9 & Cod.Or. 1871 & Panji Jaya Lengkara & Javanese & 1830 \\
\hline 10 & Cod.Or. 1881 & Malat & $\begin{array}{c}\text { Javanese- } \\
\text { Balinese }\end{array}$ & $\begin{array}{l}\text { 18th-19th } \\
\text { century }\end{array}$ \\
\hline 11 & Cod.Or. 1935 & $\begin{array}{c}\text { Hikayat Andaken } \\
\text { Penurat }\end{array}$ & Malay & 1825 \\
\hline 12 & Cod.Or. 1965 & Syair Ken Tambuhan & Malay & $\begin{array}{l}\text { 18th-19th } \\
\text { century }\end{array}$ \\
\hline 13 & Cod.Or. 1997 & Syair Ken Tambuhan & Malay & 1840 \\
\hline 14 & Cod.Or. 1998 & $\begin{array}{l}\text { Syair Undakan } \\
\text { Agung Udaya }\end{array}$ & Malay & $\begin{array}{l}\text { 18th-19th } \\
\text { century }\end{array}$ \\
\hline 15 & Cod.Or. 2005 & $\begin{array}{c}\text { Hikayat Panji } \\
\text { Susupan Mesa Kelana } \\
\text { Asmarapati }\end{array}$ & Malay & $\begin{array}{l}\text { 18th-19th } \\
\text { century }\end{array}$ \\
\hline 16 & Cod.Or. 2019 & Panji Paniba & Javanese & 1816 \\
\hline 17 & Cod.Or. 2060 & Panji Ngron Akung & Javanese & 1801 \\
\hline 18 & Cod.Or. 2061 & Malat & Javanese & $\begin{array}{l}\text { 18th-19th } \\
\text { century }\end{array}$ \\
\hline 19 & Cod.Or. 2138 (2) & Panji Ngron Akung & Javanese & 1823 \\
\hline 20 & Cod.Or. 2214 & Malat & $\begin{array}{l}\text { Javanese- } \\
\text { Balinese }\end{array}$ & $\begin{array}{c}\text { 18th-19th } \\
\text { century }\end{array}$ \\
\hline 21 & Cod.Or. 2276 (h) & $\begin{array}{l}\text { Hikayat Jawa Ratu } \\
\text { Emas Bunga Rampai }\end{array}$ & Malay. & 19th century \\
\hline 22 & Cod.Or. 2280 & Malat & Javanese & 19th century \\
\hline
\end{tabular}




\begin{tabular}{|c|c|c|c|c|}
\hline & $\begin{array}{l}\text { Codex number } \\
\text { (Cod.Or.) }\end{array}$ & Title & Language & Date \\
\hline 23 & Cod.Or. 3120 & Malat & Javanese & $\begin{array}{l}\text { 18th-19th } \\
\text { century }\end{array}$ \\
\hline 24 & Cod.Or. 3172 & [Panji romance] & Javanese & 19th century \\
\hline 25 & Cod.Or. 3192 (1) & Undakan Pangrus & $\begin{array}{l}\text { Javanese- } \\
\text { Balinese }\end{array}$ & 1824 \\
\hline 26 & Cod.Or. 3209 & $\begin{array}{c}\text { Hikayat Cekel } \\
\text { Waneng Pati }\end{array}$ & Malay & ca. 1800 \\
\hline 27 & Cod.Or. 3216a-b & $\begin{array}{c}\text { [Fragments of a Panji } \\
\text { story] }\end{array}$ & Malay & ca. 1831 \\
\hline 28 & Cod.Or. 3235 & [Panji story] & Malay & 19th century \\
\hline 29 & Cod.Or. 3236 & $\begin{array}{c}\text { Hikayat Cekel Wanen } \\
\text { Pati }\end{array}$ & Malay & 19th century \\
\hline 30 & Cod.Or. 3237 (1) & $\begin{array}{l}\text { Hikayat Panji } \\
\text { Semirang }\end{array}$ & Malay & ca. 1860 \\
\hline 31 & Cod.Or. 3238 & $\begin{array}{l}\text { [Fragments of Panji } \\
\text { stories] }\end{array}$ & Malay & 19th century \\
\hline 32 & Cod.Or. 3242 & $\begin{array}{l}\text { Hikayat Kuda } \\
\text { Semirang }\end{array}$ & Malay & 1829 \\
\hline 33 & Cod.Or. 3243 & $\begin{array}{l}\text { Hikayat Dewa } \\
\text { Asmara Jaya }\end{array}$ & Malay & 19th century \\
\hline 34 & Cod.Or. 3251 & $\begin{array}{l}\text { Hikayat Mesa Urip } \\
\text { Panji Jaya Lelana }\end{array}$ & Malay & 1818 \\
\hline 35 & Cod.Or. 3252 & Cerita Panji & Malay & 19th century \\
\hline 36 & Cod.Or. 3253 & Cerita Panji & Malay & 1868 \\
\hline 37 & Cod.Or. 3254 & $\begin{array}{l}\text { Hikayat Dewa } \\
\text { Asmara jaya }\end{array}$ & Malay & 19th century \\
\hline 38 & Cod.Or. 3365 & $\begin{array}{l}\text { Hikayat Kuda } \\
\text { Semirang }\end{array}$ & Malay & 19th century \\
\hline 39 & Cod.Or. 3367 & $\begin{array}{c}\text { Cerita Wayang } \\
\text { Kinudang } \\
\end{array}$ & Malay & 1875 \\
\hline 40 & Cod.Or. 3373 & Syair Panji & Malay & 19th century \\
\hline 41 & Cod.Or. 3375 & Syair Ken Tambuhan & Malay & 1862 \\
\hline 42 & Cod.Or. 3378 & $\begin{array}{c}\text { Hikayat Cekel } \\
\text { Waneng Pati }\end{array}$ & Malay & 19th century \\
\hline 43 & Cod.Or. 3384 & $\begin{array}{l}\text { Hikayat Panji } \\
\text { Semirang }\end{array}$ & Malay & 19th century \\
\hline 44 & Cod.Or. 3585 & Malat & $\begin{array}{l}\text { Javanese- } \\
\text { Balinese }\end{array}$ & 19th century \\
\hline 45 & Cod.Or. 3586 & Undakan Waseng Sari & $\begin{array}{l}\text { Javanese- } \\
\text { Balinese }\end{array}$ & $\begin{array}{l}\text { 18th-19th } \\
\text { century }\end{array}$ \\
\hline
\end{tabular}




\begin{tabular}{|c|c|c|c|c|}
\hline & $\begin{array}{l}\text { Codex number } \\
\text { (Cod.Or.) }\end{array}$ & Title & Language & Date \\
\hline 46 & Cod.Or. 3587 & Mantri Wadak & $\begin{array}{c}\text { Javanese- } \\
\text { Balinese }\end{array}$ & $\begin{array}{l}\text { 18th-19th } \\
\text { century }\end{array}$ \\
\hline 47 & Cod.Or. 3592 & Singa Brahmara & $\begin{array}{c}\text { Javanese- } \\
\text { Balinese }\end{array}$ & $\begin{array}{l}\text { 18th-19th } \\
\text { century }\end{array}$ \\
\hline 48 & Cod.Or. 3593 & $\begin{array}{c}\text { Datu Daha or } \\
\text { Cilinaya }\end{array}$ & Sasak & $\begin{array}{l}\text { 18th-19th } \\
\text { century }\end{array}$ \\
\hline 49 & Cod.Or. 3595 & Malat & $\begin{array}{c}\text { Javanese- } \\
\text { Balinese }\end{array}$ & $\begin{array}{c}\text { 18th-19th } \\
\text { century }\end{array}$ \\
\hline 50 & Cod.Or. 3597 & Malat & $\begin{array}{c}\text { Javanese- } \\
\text { Balinese }\end{array}$ & $\begin{array}{l}\text { 18th-19th } \\
\text { century }\end{array}$ \\
\hline 51 & Cod.Or. 3604 & Malat & $\begin{array}{c}\text { Javanese- } \\
\text { Balinese }\end{array}$ & $\begin{array}{l}\text { 18th-19th } \\
\text { century }\end{array}$ \\
\hline 52 & Cod.Or. 3610 & Wangbang Wideya & $\begin{array}{c}\text { Javanese- } \\
\text { Balinese }\end{array}$ & $\begin{array}{l}\text { 18th-19th } \\
\text { century }\end{array}$ \\
\hline 53 & Cod.Or. 3627 & Malat Kung & $\begin{array}{c}\text { Javanese- } \\
\text { Balinese }\end{array}$ & $\begin{array}{c}\text { 18th-19th } \\
\text { century }\end{array}$ \\
\hline 54 & Cod.Or. 3630 & Malat & $\begin{array}{c}\text { Javanese- } \\
\text { Balinese }\end{array}$ & $\begin{array}{c}\text { 18th-19th } \\
\text { century }\end{array}$ \\
\hline 55 & Cod.Or. 3633 & Wangbang Wideya & $\begin{array}{c}\text { Javanese- } \\
\text { Balinese }\end{array}$ & $\begin{array}{l}\text { 18th-19th } \\
\text { century }\end{array}$ \\
\hline 56 & Cod.Or. 3637 & [Panji, fragment] & Sasak & $\begin{array}{l}\text { 18th-19th } \\
\text { century }\end{array}$ \\
\hline 57 & Cod.Or. 3643 & Wangbang Wideya & $\begin{array}{c}\text { Javanese- } \\
\text { Balinese }\end{array}$ & $\begin{array}{l}\text { 18th-19th } \\
\text { century }\end{array}$ \\
\hline 58 & Cod.Or. 3644 & Undakan Waseng Sari & $\begin{array}{c}\text { Javanese- } \\
\text { Balinese }\end{array}$ & $\begin{array}{c}\text { 18th-19th } \\
\text { century }\end{array}$ \\
\hline 59 & Cod.Or. 3645 & Undakan Waseng Sari & $\begin{array}{c}\text { Javanese- } \\
\text { Balinese }\end{array}$ & $\begin{array}{l}\text { 18th-19th } \\
\text { century }\end{array}$ \\
\hline 60 & Cod.Or. 3647 & Wangbang Wideya & $\begin{array}{c}\text { Javanese- } \\
\text { Balinese }\end{array}$ & $\begin{array}{c}\text { 18th-19th } \\
\text { century }\end{array}$ \\
\hline 61 & Cod.Or. 3656 & Dangdang Petak & $\begin{array}{c}\text { Javanese- } \\
\text { Balinese }\end{array}$ & $\begin{array}{c}\text { 18th-19th } \\
\text { century }\end{array}$ \\
\hline 62 & Cod.Or. 3666 & Undakan Pangrus & $\begin{array}{c}\text { Javanese- } \\
\text { Balinese }\end{array}$ & $\begin{array}{l}\text { 18th-19th } \\
\text { century }\end{array}$ \\
\hline 63 & Cod.Or. 3667 & Ajar Pikatan & $\begin{array}{c}\text { Javanese- } \\
\text { Balinese }\end{array}$ & $\begin{array}{c}\text { 18th-19th } \\
\text { century }\end{array}$ \\
\hline 64 & Cod.Or. 3670 & Malat & $\begin{array}{c}\text { Javanese- } \\
\text { Balinese }\end{array}$ & $\begin{array}{l}\text { 18th-19th } \\
\text { century }\end{array}$ \\
\hline 65 & Cod.Or. 3701 & Satwa Pakang Baras & Balinese & $\begin{array}{l}\text { 18th-19th } \\
\text { century }\end{array}$ \\
\hline
\end{tabular}




\begin{tabular}{|c|c|c|c|c|}
\hline & $\begin{array}{c}\text { Codex number } \\
\text { (Cod.Or.) }\end{array}$ & Title & Language & Date \\
\hline 66 & Cod.Or. 3705 & Waseng Sari & $\begin{array}{l}\text { Javanese- } \\
\text { Balinese }\end{array}$ & $\begin{array}{l}\text { 18th-19th } \\
\text { century }\end{array}$ \\
\hline 67 & Cod.Or. 3710 & Malat & $\begin{array}{c}\text { Javanese- } \\
\text { Balinese }\end{array}$ & $\begin{array}{l}\text { 18th-19th } \\
\text { century }\end{array}$ \\
\hline 68 & Cod.Or. 3714 & Malat & $\begin{array}{l}\text { Javanese- } \\
\text { Balinese }\end{array}$ & $\begin{array}{l}\text { 18th-19th } \\
\text { century }\end{array}$ \\
\hline 69 & Cod.Or. 3715 & Wangbang Wideya & $\begin{array}{l}\text { Javanese- } \\
\text { Balinese }\end{array}$ & 1765 \\
\hline 70 & Cod.Or. 3720 & Mantri Wadak & $\begin{array}{l}\text { Javanese- } \\
\text { Balinese }\end{array}$ & $\begin{array}{l}\text { 18th-19th } \\
\text { century }\end{array}$ \\
\hline 71 & Cod.Or. 3721 & Malat & $\begin{array}{l}\text { Javanese- } \\
\text { Balinese }\end{array}$ & 1725 \\
\hline 72 & Cod.Or. 3726 & Swara Wedana/Malat & $\begin{array}{l}\text { Javanese- } \\
\text { Balinese }\end{array}$ & $\begin{array}{l}\text { 18th-19th } \\
\text { century }\end{array}$ \\
\hline 73 & Cod.Or. 3733 & Undakan Pangrus & $\begin{array}{l}\text { Javanese- } \\
\text { Balinese }\end{array}$ & $\begin{array}{l}\text { 18th-19th } \\
\text { century }\end{array}$ \\
\hline 74 & Cod.Or. 3737 & Malat & $\begin{array}{l}\text { Javanese- } \\
\text { Balinese }\end{array}$ & $\begin{array}{l}\text { 18th-19th } \\
\text { century }\end{array}$ \\
\hline 75 & Cod.Or. 3752 & Undakan Pangrus & $\begin{array}{l}\text { Javanese- } \\
\text { Balinese }\end{array}$ & $\begin{array}{l}\text { 18th-19th } \\
\text { century }\end{array}$ \\
\hline 76 & Cod.Or. 3765 & $\begin{array}{c}\text { Datu Daha or } \\
\text { Cilinaya }\end{array}$ & Sasak & $\begin{array}{l}\text { 18th-19th } \\
\text { century }\end{array}$ \\
\hline 77 & Cod.Or. 3770 & Undakan Pangrus & $\begin{array}{c}\text { Javanese- } \\
\text { Balinese }\end{array}$ & $\begin{array}{l}\text { 18th-19th } \\
\text { century }\end{array}$ \\
\hline 78 & Cod.Or. 3779 & Wangbang Wideya & $\begin{array}{l}\text { Javanese- } \\
\text { Balinese }\end{array}$ & $\begin{array}{l}\text { 18th-19th } \\
\text { century }\end{array}$ \\
\hline 79 & Cod.Or. 3785 & Malat & $\begin{array}{l}\text { Javanese- } \\
\text { Balinese }\end{array}$ & $\begin{array}{l}\text { 18th-19th } \\
\text { century }\end{array}$ \\
\hline 80 & Cod.Or. 3800 & Malat & $\begin{array}{c}\text { Javanese- } \\
\text { Balinese }\end{array}$ & $\begin{array}{l}\text { 18th-19th } \\
\text { century }\end{array}$ \\
\hline 81 & Cod.Or. 3812 & Pakang Raras & Balinese & $\begin{array}{l}\text { 18th-19th } \\
\text { century }\end{array}$ \\
\hline 82 & Cod.Or. 3818 & Dangdang Ireng & $\begin{array}{l}\text { Javanese- } \\
\text { Balinese }\end{array}$ & $\begin{array}{l}\text { 18th-19th } \\
\text { century }\end{array}$ \\
\hline 83 & Cod.Or. 3841 (XII) & Malat Kung & $\begin{array}{l}\text { Javanese- } \\
\text { Balinese }\end{array}$ & $\begin{array}{l}\text { 18th-19th } \\
\text { century }\end{array}$ \\
\hline 84 & Cod.Or. 3871 & Malat & $\begin{array}{l}\text { Javanese- } \\
\text { Balinese }\end{array}$ & ca.1885 \\
\hline 85 & Cod.Or. 3911 & Gunakaya & Balinese & 19th century \\
\hline 86 & Cod.Or. 3914 (1) & Pakang Raras & Balinese & 19th century \\
\hline
\end{tabular}




\begin{tabular}{|c|c|c|c|c|}
\hline & $\begin{array}{l}\text { Codex number } \\
\text { (Cod.Or.) }\end{array}$ & Title & Language & Date \\
\hline 87 & Cod.Or. 3914 (2) & Undakan Pangrus & $\begin{array}{c}\text { Javanese- } \\
\text { Balinese }\end{array}$ & 19th century \\
\hline 88 & Cod.Or. 3919 (1) & Malat & $\begin{array}{c}\text { Javanese- } \\
\text { Balinese }\end{array}$ & 19th century \\
\hline 89 & Cod.Or. 3952 (1) & Mantri Jawa & $\begin{array}{c}\text { Javanese- } \\
\text { Balinese }\end{array}$ & 19th century \\
\hline 90 & Cod.Or. 3953 (7) & Undakan Pangrus & $\begin{array}{c}\text { Javanese- } \\
\text { Balinese }\end{array}$ & 19th century \\
\hline 91 & Cod.Or. $3969(1,3)$ & Undakan Pangrus & $\begin{array}{c}\text { Javanese- } \\
\text { Balinese }\end{array}$ & 19th century \\
\hline 92 & Cod.Or. 4010 & Ajar Pikatan & $\begin{array}{c}\text { Javanese- } \\
\text { Balinese }\end{array}$ & 19th century \\
\hline 93 & Cod.Or. 4175 & Dangdang Ireng & $\begin{array}{c}\text { Javanese- } \\
\text { Balinese }\end{array}$ & 19th century \\
\hline 94 & Cod.Or. 4176 & Dangdang Ireng & $\begin{array}{c}\text { Javanese- } \\
\text { Balinese }\end{array}$ & 19th century \\
\hline 95 & Cod.Or. 4177 & Dangdang Petak & $\begin{array}{c}\text { Javanese- } \\
\text { Balinese }\end{array}$ & 19th century \\
\hline 96 & Cod.Or. 4178 & Dangdang Petak & $\begin{array}{l}\text { Javanese- } \\
\text { Balinese }\end{array}$ & 19th century \\
\hline 97 & Cod.Or. 4179 & Dangdang Petak & $\begin{array}{c}\text { Javanese- } \\
\text { Balinese }\end{array}$ & 19th century \\
\hline 98 & Cod.Or. 4180 & Dangdang Petak & $\begin{array}{c}\text { Javanese- } \\
\text { Balinese }\end{array}$ & 19th century \\
\hline 99 & Cod.Or. 4189 & $\begin{array}{c}\text { Datu Daha or } \\
\text { Cinilaya }\end{array}$ & Sasak & 19th century \\
\hline 100 & Cod.Or. 4190 & Datu Daha & Sasak & 19th century \\
\hline 101 & Cod.Or. 4302 & Malat Kung & $\begin{array}{c}\text { Javanese- } \\
\text { Balinese }\end{array}$ & 19th century \\
\hline 102 & Cod.Or. 4303 & Malat & $\begin{array}{c}\text { Javanese- } \\
\text { Balinese }\end{array}$ & 19th century \\
\hline 103 & Cod.Or. 4304 & Malat & $\begin{array}{c}\text { Javanese- } \\
\text { Balinese }\end{array}$ & 19th century \\
\hline 104 & Cod.Or. 4305 & Malat & $\begin{array}{c}\text { Javanese- } \\
\text { Balinese }\end{array}$ & 19th century \\
\hline 105 & Cod.Or. 4306 & Malat & $\begin{array}{c}\text { Javanese- } \\
\text { Balinese }\end{array}$ & 19th century \\
\hline 106 & Cod.Or. 4307 & Malat & $\begin{array}{c}\text { Javanese- } \\
\text { Balinese }\end{array}$ & 19th century \\
\hline 107 & Cod.Or. 4308 & Malat & $\begin{array}{c}\text { Javanese- } \\
\text { Balinese }\end{array}$ & 19th century \\
\hline
\end{tabular}




\begin{tabular}{|c|c|c|c|c|}
\hline & $\begin{array}{l}\text { Codex number } \\
\text { (Cod.Or.) }\end{array}$ & Title & Language & Date \\
\hline 108 & Cod.Or. 4309 & Malat & $\begin{array}{l}\text { Javanese- } \\
\text { Balinese }\end{array}$ & 19th century \\
\hline 109 & Cod.Or. 4310 & Malat & $\begin{array}{l}\text { Javanese- } \\
\text { Balinese }\end{array}$ & 19th century \\
\hline 110 & Cod.Or. 4311 & Malat & $\begin{array}{l}\text { Javanese- } \\
\text { Balinese }\end{array}$ & 19th century \\
\hline 111 & Cod.Or. 4312 & Malat & $\begin{array}{l}\text { Javanese- } \\
\text { Balinese }\end{array}$ & 19th century \\
\hline 112 & Cod.Or. 4313 & Malat & $\begin{array}{l}\text { Javanese- } \\
\text { Balinese }\end{array}$ & 19th century \\
\hline 113 & Cod.Or. 4314 & Malat & $\begin{array}{l}\text { Javanese- } \\
\text { Balinese }\end{array}$ & 19th century \\
\hline 114 & Cod.Or. 4315 & Malat & $\begin{array}{l}\text { Javanese- } \\
\text { Balinese }\end{array}$ & 19th century \\
\hline 115 & Cod.Or. 4316 & Malat & $\begin{array}{l}\text { Javanese- } \\
\text { Balinese }\end{array}$ & 19th century \\
\hline 116 & Cod.Or. 4317 & Malat & $\begin{array}{l}\text { Javanese- } \\
\text { Balinese }\end{array}$ & 19th century \\
\hline 117 & Cod.Or. 4318 & Malat & $\begin{array}{l}\text { Javanese- } \\
\text { Balinese }\end{array}$ & 19th century \\
\hline 118 & Cod.Or. 4319 & Malat & $\begin{array}{c}\text { Javanese- } \\
\text { Balinese }\end{array}$ & 19th century \\
\hline 119 & Cod.Or. 4320 & Malat & $\begin{array}{l}\text { Javanese- } \\
\text { Balinese }\end{array}$ & 19th century \\
\hline 120 & Cod.Or. 4321 & Malat & $\begin{array}{l}\text { Javanese- } \\
\text { Balinese }\end{array}$ & 19th century \\
\hline 121 & Cod.Or. 4322 & Malat & $\begin{array}{l}\text { Javanese- } \\
\text { Balinese }\end{array}$ & 19th century \\
\hline 122 & Cod.Or. 4323 & Malat & $\begin{array}{l}\text { Javanese- } \\
\text { Balinese }\end{array}$ & 19th century \\
\hline 123 & Cod.Or. 4324 & Mantri Wadak & $\begin{array}{c}\text { Javanese- } \\
\text { Balinese }\end{array}$ & 19th century \\
\hline 124 & Cod.Or. 4325 & Mantri Wadak & $\begin{array}{l}\text { Javanese- } \\
\text { Balinese }\end{array}$ & 19th century \\
\hline 125 & Cod.Or. 4329 & Marga Smara & $\begin{array}{l}\text { Javanese- } \\
\text { Balinese }\end{array}$ & 19th century \\
\hline 126 & Cod.Or. 4330 & Marga Swara & $\begin{array}{l}\text { Javanese- } \\
\text { Balinese }\end{array}$ & $\begin{array}{l}\text { 18th-19th } \\
\text { century }\end{array}$ \\
\hline 127 & Cod.Or. 4339 & Misa Gagang & Javanese & 19th century \\
\hline 128 & Cod.Or. 4359 & Pakang Raras & Balinese & 19th century \\
\hline
\end{tabular}




\begin{tabular}{|c|c|c|c|c|}
\hline & $\begin{array}{l}\text { Codex number } \\
\text { (Cod.Or.) }\end{array}$ & Title & Language & Date \\
\hline 129 & Cod.Or. 4480 (1) & $\begin{array}{c}\text { Satwa }=\text { Pakang } \\
\text { Raras }\end{array}$ & Balinese & 19th century \\
\hline 130 & Cod.Or. 4490 & Singa Brahmara & $\begin{array}{c}\text { Javanese- } \\
\text { Balinese }\end{array}$ & 19th century \\
\hline 131 & Cod.Or. 4496 & Smara Wedana & $\begin{array}{c}\text { Javanese- } \\
\text { Balinese }\end{array}$ & 1763 \\
\hline 132 & Cod.Or. 4497 & Smara Wedana & $\begin{array}{c}\text { Javanese- } \\
\text { Balinese }\end{array}$ & 18th century \\
\hline 133 & Cod.Or. 4498 & Smara Wedana & $\begin{array}{c}\text { Javanese- } \\
\text { Balinese }\end{array}$ & 1714 \\
\hline 134 & Cod.Or. 4587 & $\begin{array}{c}\text { Cilinaya or Datu } \\
\text { Daha }\end{array}$ & Sasak & 19th century \\
\hline 135 & Cod.Or. 4606 & Undakan Pangrus & $\begin{array}{c}\text { Javanese- } \\
\text { Balinese }\end{array}$ & 19th century \\
\hline 136 & Cod.Or. 4607 & Undakan Pangrus & $\begin{array}{c}\text { Javanese- } \\
\text { Balinese }\end{array}$ & 19th century \\
\hline 137 & Cod.Or. 4608 & Undakan Pangrus & $\begin{array}{c}\text { Javanese- } \\
\text { Balinese }\end{array}$ & 19th century \\
\hline 138 & Cod.Or. 4609 & Undakan Pangrus & $\begin{array}{c}\text { Javanese- } \\
\text { Balinese }\end{array}$ & 19th century \\
\hline 139 & Cod.Or. 4610 & Undakan Pangrus & $\begin{array}{c}\text { Javanese- } \\
\text { Balinese }\end{array}$ & 19th century \\
\hline 140 & Cod.Or. 4642 & Wangbang Wideya & $\begin{array}{c}\text { Javanese- } \\
\text { Balinese }\end{array}$ & 19th century \\
\hline 141 & Cod.Or. 4643 & Wangbang Wideya & $\begin{array}{c}\text { Javanese- } \\
\text { Balinese }\end{array}$ & 19th century \\
\hline 142 & Cod.Or. 4644 & Wangbang Wideya & $\begin{array}{c}\text { Javanese- } \\
\text { Balinese }\end{array}$ & 19th century \\
\hline 143 & Cod.Or. 4645 & Wangbang Wideya & $\begin{array}{c}\text { Javanese- } \\
\text { Balinese }\end{array}$ & 19th century \\
\hline 144 & Cod.Or. 4646 & Wangbang Wideya & $\begin{array}{c}\text { Javanese- } \\
\text { Balinese }\end{array}$ & 19th century \\
\hline 145 & Cod.Or. 4647 & Wangbang Wideya & $\begin{array}{c}\text { Javanese- } \\
\text { Balinese }\end{array}$ & 19th century \\
\hline 146 & Cod.Or. 4648 & Wangbang Wideya & $\begin{array}{c}\text { Javanese- } \\
\text { Balinese }\end{array}$ & 19th century \\
\hline 147 & Cod.Or. 4665 & Waseng Sari & $\begin{array}{c}\text { Javanese- } \\
\text { Balinese }\end{array}$ & 19th century \\
\hline 148 & Cod.Or. 4666 & Waseng Sari & $\begin{array}{c}\text { Javanese- } \\
\text { Balinese }\end{array}$ & 19th century \\
\hline
\end{tabular}




\begin{tabular}{|c|c|c|c|c|}
\hline & $\begin{array}{l}\text { Codex number } \\
\text { (Cod.Or.) }\end{array}$ & Title & Language & Date \\
\hline 149 & Cod.Or. 4667 & Waseng Sari & $\begin{array}{l}\text { Javanese- } \\
\text { Balinese }\end{array}$ & 19th century \\
\hline 150 & Cod.Or. 4668 & Waseng Sari & $\begin{array}{l}\text { Javanese- } \\
\text { Balinese }\end{array}$ & 19th century \\
\hline 151 & Cod.Or. 4669 & Waseng Sari & $\begin{array}{l}\text { Javanese- } \\
\text { Balinese }\end{array}$ & 19th century \\
\hline 152 & Cod.Or. 4670 & Waseng Sari & $\begin{array}{l}\text { Javanese- } \\
\text { Balinese }\end{array}$ & 19th century \\
\hline 153 & Cod.Or. 4671 & Waseng Sari & $\begin{array}{l}\text { Javanese- } \\
\text { Balinese }\end{array}$ & 19th century \\
\hline 154 & Cod.Or. 4983 & Malat & $\begin{array}{l}\text { Javanese- } \\
\text { Balinese }\end{array}$ & 1868 \\
\hline 155 & Cod.Or. 5012 & Malat & $\begin{array}{l}\text { Javanese- } \\
\text { Balinese }\end{array}$ & 19th century \\
\hline 156 & Cod.Or. 5017 & Smara Wijaya - Malat & $\begin{array}{l}\text { Javanese- } \\
\text { Balinese }\end{array}$ & 1843 \\
\hline 157 & Cod.Or. 5044 & $\begin{array}{c}\text { Indra Wismara- } \\
\text { Malat }\end{array}$ & $\begin{array}{l}\text { Javanese- } \\
\text { Balinese }\end{array}$ & 19th century \\
\hline 158 & Cod.Or. 5092 (1) & Waseng Sari & $\begin{array}{l}\text { Javanese- } \\
\text { Balinese }\end{array}$ & 19th century \\
\hline 159 & Cod.Or. 5388 & Indra Wismara & $\begin{array}{l}\text { Javanese- } \\
\text { Balinese }\end{array}$ & 19th century \\
\hline 160 & Cod.Or. 5437 & Waseng Sari & $\begin{array}{l}\text { Javanese- } \\
\text { Balinese }\end{array}$ & 19th century \\
\hline 161 & Cod.Or. 6428 & $\begin{array}{l}51 \text { lakons of wayang } \\
\text { gedog (Panji tales) }\end{array}$ & Javanese & $1898-1905$ \\
\hline 162 & Cod.Or. 6750 & Panji Ngron Akung & Javanese & 1936 \\
\hline 163 & Cod.Or. 6751 & Panji Murta Smara & Javanese & 20th century \\
\hline 164 & Cod.Or. 6752 & $\begin{array}{c}\text { Panji Jaya Lengkara } \\
\text { and Angreni }\end{array}$ & Javanese & 20th century \\
\hline 165 & Cod.Or. 6841 & $\begin{array}{c}\text { Hikayat Mesa } \\
\text { Gumitar }\end{array}$ & Malay & 1821 \\
\hline 166 & Cod.Or. 7227 & Wangbang Wideya & $\begin{array}{c}\text { Javanese- } \\
\text { Balinese }\end{array}$ & 20th century \\
\hline 167 & $\begin{array}{c}\text { Cod.Or. } 7403 \mathrm{a}, \mathrm{b} \\
(14)\end{array}$ & Hikayat suluk Panji & Sundanese & 1907 \\
\hline 168 & Cod.Or. 7785 & $\begin{array}{l}\text { Wawacan Panji } \\
\text { Asmara Ningrat }\end{array}$ & Sundanese & 19th century \\
\hline 169 & Cod.Or. 7836 (1) & $\begin{array}{c}\text { Suluk Panji } \\
\text { Brataningrat }\end{array}$ & Sundanese & 19th century \\
\hline 170 & Cod.Or. 8101 & Hikayat Cintabuhan & Acehnese & 19th century \\
\hline
\end{tabular}




\begin{tabular}{|c|c|c|c|c|}
\hline & $\begin{array}{l}\text { Codex number } \\
\text { (Cod.Or.) }\end{array}$ & Title & Language & Date \\
\hline 171 & Cod.Or. 8102 & Hikayat Cintabuhan & Acehnese & 1873 \\
\hline 172 & Cod.Or. 8103 & Hikayat Cintabuhan & Acehnese & 1893 \\
\hline 173 & Cod.Or. 8104 & Hikayat Cintabuhan & Acehnese & 20th century \\
\hline 174 & Cod.Or. $8401 \mathrm{f}$ & $\begin{array}{c}\text { Hikayat Panji } \\
\text { Susupan Mesa Kelana } \\
\text { Asmarapati }\end{array}$ & Malay & 20th century \\
\hline 175 & Cod.Or. 8476 & Malat & $\begin{array}{l}\text { Javanese- } \\
\text { Balinese }\end{array}$ & $\begin{array}{l}\text { 19th-20th } \\
\text { century }\end{array}$ \\
\hline 176 & Cod.Or. 8941 & Panji Priyembada & Javanese & 1749 \\
\hline 177 & Cod.Or. 9006 & Malat & $\begin{array}{c}\text { Javanese- } \\
\text { Balinese }\end{array}$ & $\begin{array}{c}\text { 19th-20th } \\
\text { century }\end{array}$ \\
\hline 178 & Cod.Or. 9369 & Marut Smara & $\begin{array}{c}\text { Javanese- } \\
\text { Balinese }\end{array}$ & 20th century \\
\hline 179 & Cod.Or. 9669 & Kuda Rawi Sandi & $\begin{array}{c}\text { Javanese- } \\
\text { Balinese }\end{array}$ & 20th century \\
\hline 180 & Cod.Or. 9758 & Lembu Raga & $\begin{array}{c}\text { Javanese- } \\
\text { Balinese }\end{array}$ & 20th century \\
\hline 181 & Cod.Or. 10.097 & Malat Parikan & Balinese & 20th century \\
\hline 182 & Cod.Or. 10.156 & Malat Parikan & Balinese & 20th century \\
\hline 183 & Cod.Or. 10.217 & $\begin{array}{c}\text { Geguritan Mantri } \\
\text { Jawa }\end{array}$ & Balinese & 20th century \\
\hline 184 & Cod.Or. 10.253 & $\begin{array}{c}\text { Geguritan Panji } \\
\text { Semirang }\end{array}$ & $\begin{array}{c}\text { Javanese- } \\
\text { Balinese }\end{array}$ & 20th century \\
\hline 185 & Cod.Or. 10.304 & $\begin{array}{c}\text { Cilinaya or Datu } \\
\text { Daha }\end{array}$ & Sasak & 20th century \\
\hline 186 & Cod.Or. 10.350 & $\begin{array}{c}\text { Cilinaya or Datu } \\
\text { Daha }\end{array}$ & Sasak & 20th century \\
\hline 187 & Cod.Or. 10.470 & Wangbang Wideya & $\begin{array}{c}\text { Javanese- } \\
\text { Balinese }\end{array}$ & 20th century \\
\hline 188 & Cod.Or. 10.472 & Indra Wismara & $\begin{array}{c}\text { Javanese- } \\
\text { Balinese }\end{array}$ & 20th century \\
\hline 189 & Cod.Or. 10.482 & Dangdang Ireng & $\begin{array}{c}\text { Javanese- } \\
\text { Balinese }\end{array}$ & 20th century \\
\hline 190 & Cod.Or. 10.488 & Singa Brahmara & $\begin{array}{c}\text { Javanese- } \\
\text { Balinese }\end{array}$ & 20th century \\
\hline 191 & Cod.Or. 10.489 & Undakan Pangrus & $\begin{array}{c}\text { Javanese- } \\
\text { Balinese }\end{array}$ & 20th century \\
\hline 192 & Cod.Or. 10.503 & Smara Wedana & $\begin{array}{c}\text { Javanese- } \\
\text { Balinese }\end{array}$ & 20th century \\
\hline 193 & Cod.Or. 10.615 & Cili Naya & $\begin{array}{c}\text { Javanese- } \\
\text { Balinese }\end{array}$ & 20th century \\
\hline
\end{tabular}




\begin{tabular}{|c|c|c|c|c|}
\hline & $\begin{array}{l}\text { Codex number } \\
\text { (Cod.Or.) }\end{array}$ & Title & Language & Date \\
\hline 194 & Cod.Or. 10.878 (1) & Malat & $\begin{array}{l}\text { Javanese- } \\
\text { Balinese }\end{array}$ & 20th century \\
\hline 195 & Cod.Or. 10.878 (2) & Malat & $\begin{array}{l}\text { Javanese- } \\
\text { Balinese }\end{array}$ & 20th century \\
\hline 196 & Cod.Or. 10.934 & $\begin{array}{c}\text { Panji Jaka Kembang } \\
\text { Kuning }\end{array}$ & Javanese & $\begin{array}{l}\text { 19th-20th } \\
\text { century }\end{array}$ \\
\hline 197 & Cod.Or. 10.988 & Jaya Lengkara & Javanese & 1860 \\
\hline 198 & Cod.Or. 11.168 & Nagari Arta Warsa & $\begin{array}{l}\text { Javanese- } \\
\text { Balinese }\end{array}$ & 1940 \\
\hline 199 & Cod.Or. 12.959 & Undakan Pangrus & $\begin{array}{l}\text { Javanese- } \\
\text { Balinese }\end{array}$ & 20th century \\
\hline 200 & Cod.Or. 13.301 & Wedanta Wiwaha & $\begin{array}{c}\text { Javanese- } \\
\text { Balinese }\end{array}$ & 1973 \\
\hline 201 & Cod.Or. 13.303 & Panji Marga Swara & $\begin{array}{l}\text { Javanese- } \\
\text { Balinese }\end{array}$ & 1973 \\
\hline 202 & Cod.Or. 13.358 & Undakan Pangrus & $\begin{array}{l}\text { Javanese- } \\
\text { Balinese }\end{array}$ & 20th century \\
\hline 203 & Cod.Or. 13.448 & Kusuma Wijaya & $\begin{array}{c}\text { Javanese- } \\
\text { Balinese }\end{array}$ & 20th century \\
\hline 204 & Cod.Or. 13.515 & Undakan Pangrus & $\begin{array}{l}\text { Javanese- } \\
\text { Balinese }\end{array}$ & 20th century \\
\hline 205 & Cod.Or. 13.530 & Malat Kung & $\begin{array}{l}\text { Javanese- } \\
\text { Balinese }\end{array}$ & 1973 \\
\hline 206 & Cod.Or. 13.540 & Wangbang Turida & $\begin{array}{c}\text { Javanese- } \\
\text { Balinese }\end{array}$ & 20th century \\
\hline 207 & Cod.Or. 13.571 & Malat Kung & $\begin{array}{l}\text { Javanese- } \\
\text { Balinese }\end{array}$ & 20th century \\
\hline 208 & Cod.Or. 13.658 & Wijaya Smara & $\begin{array}{c}\text { Javanese- } \\
\text { Balinese }\end{array}$ & 20th century \\
\hline 209 & Cod.Or. 13.688 & Rawi Sandi & $\begin{array}{l}\text { Javanese- } \\
\text { Balinese }\end{array}$ & 20th century \\
\hline 210 & Cod.Or. 13.732 & Malat Rasmi & $\begin{array}{c}\text { Javanese- } \\
\text { Balinese }\end{array}$ & 20th century \\
\hline 211 & Cod.Or. 13.737 & Malat Rasmi & $\begin{array}{l}\text { Javanese- } \\
\text { Balinese }\end{array}$ & 20th century \\
\hline 212 & Cod.Or. 13.752 & Dangdang Petak & $\begin{array}{l}\text { Javanese- } \\
\text { Balinese }\end{array}$ & 20th century \\
\hline 213 & Cod.Or. 13.790 & Malat Rasmi & $\begin{array}{l}\text { Javanese- } \\
\text { Balinese }\end{array}$ & 20th century \\
\hline 214 & Cod.Or. 13.792 & Widari Smara & $\begin{array}{l}\text { Javanese- } \\
\text { Balinese }\end{array}$ & 20th century \\
\hline
\end{tabular}




\begin{tabular}{|c|c|c|c|c|}
\hline & $\begin{array}{l}\text { Codex number } \\
\text { (Cod.Or.) }\end{array}$ & Title & Language & Date \\
\hline 215 & Cod.Or. 13.816 & Malat Rasmi & $\begin{array}{c}\text { Javanese- } \\
\text { Balinese }\end{array}$ & 20th century \\
\hline 216 & Cod.Or. 13.921 & Wangbang Turida & $\begin{array}{l}\text { Javanese- } \\
\text { Balinese }\end{array}$ & 20th century \\
\hline 217 & Cod.Or. 14.080 & Syair Ken Tambuhan & Malay & 1850 \\
\hline 218 & Cod.Or. 14.433 & $\begin{array}{c}\text { Hikayat Mesa } \\
\text { Gumitar }\end{array}$ & Malay & 1977 \\
\hline 219 & Cod.Or. 14.717 & Dangdang Petak & $\begin{array}{c}\text { Javanese- } \\
\text { Balinese }\end{array}$ & 20th century \\
\hline 220 & Cod.Or. 14.792 & Panji Marga Swara & $\begin{array}{c}\text { Javanese- } \\
\text { Balinese }\end{array}$ & 20th century \\
\hline 221 & Cod.Or. 18.152 & Satwa Bakang Paras & Balinese & 20th century \\
\hline 222 & Cod.Or. 18.157 & $\begin{array}{l}\text { Kidung Panji Malat } \\
\text { Rasmin }\end{array}$ & Javanese & 20th century \\
\hline 223 & Cod.Or. 18.253 & $\begin{array}{c}\text { Kidung Panji Malat } \\
\text { Rasmin }\end{array}$ & Javanese & 20th century \\
\hline 224 & Cod.Or. 18.803 & Satua Pakang Raras & Balinese & 20th century \\
\hline 225 & Cod.Or. 19.141 & $\begin{array}{c}\text { Kidung Panji Malat } \\
\text { Rasmi }\end{array}$ & Javanese & 1984 \\
\hline 226 & Cod.Or. 19.420 & $\begin{array}{c}\text { Kidung Panji-Sakti- } \\
\text { Wijaya }\end{array}$ & Javanese & 20th century \\
\hline 227 & Cod.Or. 19.454 & $\begin{array}{c}\text { Kidung Panji-Sakti } \\
\text { Wijaya } \\
\end{array}$ & Balinese & 20th century \\
\hline 228 & Cod.Or. 19.521 & $\begin{array}{c}\text { Gaguritan Panji } \\
\text { Semirang }\end{array}$ & Balinese & 20th century \\
\hline 229 & Cod.Or. 19.5220 & $\begin{array}{l}\text { Gaguritan Panji } \\
\text { Semirang }\end{array}$ & Balinese & 20th century \\
\hline 230 & Cod.Or. 19.550 & $\begin{array}{c}\text { Kidung Panji Wijaya- } \\
\text { krama. }\end{array}$ & Javanese & 20th century \\
\hline 231 & Cod.Or. 19.557 & $\begin{array}{c}\text { Kaputusan Panji } \\
\text { Rawit. }\end{array}$ & Javanese & 20th century \\
\hline 232 & Cod.Or. 19.586 & $\begin{array}{l}\text { Kidung Panji } \\
\text { Sumirang. }\end{array}$ & Javanese & 20th century \\
\hline 233 & Cod.Or. 19.638 & $\begin{array}{c}\text { Paparikan Panji } \\
\text { Semirang }\end{array}$ & Balinese & 20th century \\
\hline 234 & Cod.Or. 20.017 & $\begin{array}{c}\text { Kidung Panji-Sakti- } \\
\text { Wijaya }\end{array}$ & Javanese & 20th century \\
\hline 235 & Cod.Or. 20.124 & $\begin{array}{c}\text { Kidung Panji-Sakti } \\
\text { Wijaya }\end{array}$ & Balinese & 20th century \\
\hline 236 & Cod.Or. 20.297 & $\begin{array}{c}\text { Gaguritan Panji } \\
\text { Semirang }\end{array}$ & Balinese & 20th century \\
\hline
\end{tabular}




\begin{tabular}{|c|c|c|c|c|}
\hline & $\begin{array}{l}\text { Codex number } \\
\text { (Cod.Or.) }\end{array}$ & Title & Language & Date \\
\hline 237 & Cod.Or. 20.303 & $\begin{array}{c}\text { Gaguritan Panji } \\
\text { Semirang }\end{array}$ & Balinese & 20th century \\
\hline 238 & Cod.Or. 20.326 & $\begin{array}{l}\text { Kaputusan Panji } \\
\text { Rawit. }\end{array}$ & Javanese & 20th century \\
\hline 239 & Cod.Or. 20.332 & $\begin{array}{l}\text { Kidung Panji Wijaya- } \\
\text { krama. }\end{array}$ & Javanese & 20th century \\
\hline 240 & Cod.Or. 20.346 & $\begin{array}{l}\text { Kidung Panji } \\
\text { Sumirang. }\end{array}$ & Javanese & 20th century \\
\hline 241 & Cod.Or. 20.385 & $\begin{array}{l}\text { Paparikan Panji } \\
\text { Semirang }\end{array}$ & Balinese & 20th century \\
\hline 242 & Cod.Or. 20.441 & $\begin{array}{c}\text { Kidung Panji Malat } \\
\text { Rasmin }\end{array}$ & Javanese & 20th century \\
\hline 243 & Cod.Or. 22.647 & Malat Panji Rasmin & Javanese & $\begin{array}{l}\text { 19th-20th } \\
\text { century }\end{array}$ \\
\hline 244 & Cod.Or. 22.649 & Malat Panji Semirang & Javanese & $\begin{array}{l}\text { 19th-20th } \\
\text { century }\end{array}$ \\
\hline 245 & Cod.Or. 22.948 & $\begin{array}{c}\text { Serat Panji Wulan } \\
\text { Tumanggal }\end{array}$ & Javanese & 1889 \\
\hline 246 & Cod.Or. 23.545 & $\begin{array}{l}\text { Geguritan Panji } \\
\text { Malat Rasmi }\end{array}$ & Balinese & $\begin{array}{l}\text { 19th-20th } \\
\text { century }\end{array}$ \\
\hline 247 & Cod.Or. 23.799 & Panji Sakti Wijaya & Javanese & 1904 \\
\hline 248 & Cod.Or. 23.864 & $\begin{array}{c}\text { Parikan Candra } \\
\text { Kirana, Panji } \\
\text { Semirang }\end{array}$ & Balinese & $\begin{array}{l}\text { 19th-20th } \\
\text { century }\end{array}$ \\
\hline 249 & Cod.Or. 24.789 & $\begin{array}{l}\text { Geguritan Panji } \\
\text { Semirang }\end{array}$ & Balinese & 20th century \\
\hline 250 & Cod.Or. 24.828 & $\begin{array}{c}\text { Kidung Panji Malat } \\
\text { Rasmi }\end{array}$ & Javanese & 20th century \\
\hline 251 & Cod.Or. 24.892 & Geguritan Panji & Balinese & 20th century \\
\hline 252 & Cod.Or. 24.984 & $\begin{array}{c}\text { Kidung Panji Malat } \\
\text { Rasmi }\end{array}$ & Javanese & 20th century \\
\hline 253 & Cod.Or. 25.959 & Kidung Panjimarga & Balinese & 20th century \\
\hline 254 & Cod.Or. 26.275 & $\begin{array}{c}\text { Kidung Panji Malat } \\
\text { Rasmi, }\end{array}$ & Javanese & 2001 \\
\hline 255 & Cod.Or. 26.435 & $\begin{array}{l}\text { Gaguritan Panji } \\
\text { Semirang. }\end{array}$ & Balinese & 20th century \\
\hline 256 & Cod.Or. 26.468 & Gaguritan Panji & Balinese & 20th century \\
\hline 257 & NBG Boeg 85 & Cékéléq & Buginese & са. 1860 \\
\hline 258 & NBG Boeg 86 & Cékéléq & Buginese & ca. 1860 \\
\hline
\end{tabular}




\begin{tabular}{|c|c|c|c|c|}
\hline & $\begin{array}{c}\text { Codex number } \\
\text { (Cod.Or.) }\end{array}$ & Title & Language & Date \\
\hline 259 & K1. 1 & $\begin{array}{c}\text { Hikayat Mesa } \\
\text { Kagungan Seri Panji } \\
\text { Wila Kesuma }\end{array}$ & Malay & 1864 \\
\hline 260 & K1. 12 & $\begin{array}{c}\text { Hikayat Mesa Perabu } \\
\text { Jaya }\end{array}$ & Malay & 1863 \\
\hline 261 & K1. 13 & $\begin{array}{c}\text { Hikayat Mesa Perabu } \\
\text { Jaya }\end{array}$ & Malay & 1850 \\
\hline 262 & K1. 44 II & Cerita Panji & Malay & 1822 \\
\hline 263 & K1. 149 & Syair Ken Tambuhan & Malay & 1862 \\
\hline
\end{tabular}

